\title{
ELEMENTOS DE INNOVACIÓN EN HOTELES. MAZATLÁN, SINALOA, MÉXICO
}

\section{ELEMENTS OF INNOVATION IN HOTELS. MAZATLAN, SINALOA, MEXICO}

Ana maría Larrañaga Núñez ${ }^{1}$

Martín León Santiesteban²

1. Profesora del Departamento de Ciencias Sociales y Humanidades de la Universidad de Occidente, Culiacán (México). E-mail: amlarranaga2607@gmail.com

2. Doctor en Gestión del Turismo. Docente de la Maestría de Gestión y Políticas Públicas de la Universidad de Occidente, Culiacán (México). E-mail: martin.leon@udo.mx

Citación sugerida:

Larrañaga Núñez, A.M. y León Santiesteban, M. (2017). Elementos de innovación en hoteles. Mazatlán, Sinaloa, México. 3C Empresa: investigación y pensamiento crítico, 6(2), 20-32. DOI: <http://dx.doi.org/10.17993/3cemp.2017.060230.20-32/>. 


\section{RESUMEN}

Este artículo contiene hallazgos de investigación sobre elementos que han contribuido en las innovaciones de procesos y organizativas como ventaja competitiva de hoteles en el destino Mazatlán, Sinaloa, México. Los factores que motivan la innovación y los obstáculos han generado innovaciones moderadas. El método mixto permitió la recolección de los datos. Los resultados del cuestionario dan cuenta que la tendencia para innovar se da en la dimensión organización del lugar de trabajo mientras los obstáculos se presentan en la dimensión de factores costo y vinculados al conocimiento en la innovación de procesos, los resultados de las entrevistas semiestructuradas dieron lugar a categorías como actividades de innovación desde lo individual y a fuerzas como la inseguridad y prácticas de sustentabilidad.

\section{ABSTRACT}

This article contains research findings on elements that have contributed to process and organizational innovations as a competitive advantage of hotels in the Mazatlán, Sinaloa, Mexico destination. The factors that motivate innovation and obstacles have generated modest innovations. The mixed method allowed data collection. The results of the questionnaire indicate that the tendency to innovate occurs in the organizational dimension of the workplace while the obstacles are presented in the dimension of cost factors and linked to knowledge in process innovation; the results of semi-structured interviews gave rise to categories such as individual innovation activities and to forces such as insecurity and sustainability practices.

\section{PALABRAS CLAVE}

Innovación de proceso, innovación organizativa, hoteles, destino.

\section{KEY WORDS}

Innovation of process, organizational innovation, hotels, destination. 


\section{INTRODUCCIÓN}

La innovación y su implementación en las empresas de hospedaje de los destinos turísticos tiene sentido como cambio y diferenciación (Weiermair, 2004) y (Nieves y Segarra-Ciprés, 2015) para enfrentar a la competencia y el riesgo de disminuir sus ventajas competitivas ante ofertas emergentes, sus propias inercias, las exigencias de los visitantes y otros factores como la inseguridad.

El objetivo de esta investigación es determinar los elementos de la innovación de procesos (IP) e innovación organizativa (IO) de los establecimientos de hotelería en el destino turístico de Mazatlán, Sinaloa, México, mediante el análisis de las dimensiones: las actividades que se realizan, los factores que motivan o los obstáculos para su implementación.

Los antecedentes de Mazatlán dan cuenta que es Municipio y cabecera del mismo nombre, se encuentra ubicado en la parte sur del estado de Sinaloa, al noroeste de la República Mexicana a 21 kilómetros del trópico de Cáncer. Es un destino turístico tradicional de sol y playa que ha tenido un comportamiento irregular en la demanda (Santamaría y Barbosa, 2008). A partir de 2005 se tiene un ciclo de crecimiento en la actividad hotelera que se manifiesta en incrementos $94.4 \%$ de llegadas de visitantes con respecto a 2015 y 6.8 puntos porcentuales de ocupación en el período 2013-2015 Secretaría de Turismo (SECTUR) federal (2015).

Las ventajas competitivas (Ritchie y Crouche, 2011) de Mazatlán se tienen en inversiones públicas en infraestructura carretera, privadas e inmobiliarios que aumentan el número de cuartos promedio, diversificación de segmentos turísticos y la certificación de dos de sus playas; las desventajas se tienen en la afectación de la imagen producto de la inseguridad y desarrollo turísticos regionales emergentes como la Riviera Nayarita (SECTUR, 2014).

\section{INNOVACIÓN}

La innovación es la conversión de ideas y conocimientos en productos, procesos o servicios mejorados para el mercado, satisfaciendo así las necesidades de los ciudadanos, empresas y administraciones públicas (Corma, 2011) y (Afuah, 1999). La Organización para la Cooperación y Desarrollo Económicos (OCDE, 2006) menciona que la innovación es la introducción de un nuevo o significativamente mejorado producto (bien o servicio) de un proceso, método de comercialización, método organizativo en las prácticas internas de la empresa, la organización del lugar de trabajo o las relaciones exteriores. Mathison, Gándara, Primera y García, (2007) señalan que la innovación es "la capacidad para transformar los procesos empresariales y crear organizaciones más competitivas, ágiles y eficaces" (p. 69). 
Hall y Williams (2008) mencionan que las características de la innovación en el turismo se relacionan con los subsectores de servicios por los consumidores finales. Entre los rasgos distintivos, señalan: la importancia del recurso humano en donde la calidad del trabajo da forma a la experiencia turística y, a factores organizacionales que son significativos siendo vinculados con cambios en la gestión, procesos, productos e innovaciones de mercado.

La importancia de la innovación en la actividad turística (Mohd y Hilmi, 2012) se presenta en la promoción y aprovechamiento de todas las ventajas y el potencial del sector, ya que da una ventaja competitiva frente a destinos. Vila, Enz y Costa (2012) y (Pivcevic y Garbinm, 2012) se refieren a la dificultad de innovar en la hotelería ante la imitación de ahí que recomiendan aprovechar los recursos para agregar valor al servicio y crear una competencia diferenciada y sostenible. Es decir, la innovación y su implementación en la hotelería y en los destinos turísticos tiene sentido como cambio (Weiermair, 2004) y (Nieves y Segarra-Ciprés, 2015) y diferenciación para enfrentar a la competencia, así como el riesgo de disminuir sus ventajas competitiva.

En relación con los factores y efectos que motivan la innovación la OCDE (2006) enfatiza que las empresas innovan por razones múltiples: sus objetivos pueden referirse a los productos, los mercados, la eficiencia, la calidad o la aptitud para aprender a introducir cambios. Weiermair, (2004) menciona tres factores que determinan el nivel y el ritmo de la innovación de forma activa en el turismo, mediante los suministros relacionados con la oferta, los impulsores de la demanda y el ritmo de la competencia.

En relación con la oferta, enfatiza Weiermair, (2004) que la disponibilidad de tecnologías ha llevado al desarrollo de nuevas habilidades y formas de organización y, nuevos materiales y servicios. Menciona que la orientación al cliente es relevante en la innovación de servicios, ya que se puede obtener información valiosa a partir de su comportamiento. Ante ello las empresas tienen que adaptarse a los cambiantes intereses y valores de sus clientes. Por último, dice que la globalización y la desregulación han incrementado la competencia, fenómeno que contribuye en la IP (sistemas de redes, de reserva y de gestión del rendimiento, etc.) en oposición a la innovación de productos que pueden ser fácilmente imitados por los competidores rivales.

En las empresas pueden presentarse obstáculos para innovar, entre ellos se encuentra el costo que implica la actividad y los factores asociados al conocimiento, además pueden tener éxito o no al tratar de alcanzar los objetivos que se habían fijado, a su vez las innovaciones pueden tener efectos inesperados o adicionales con relación a los que se habían sustituido con su introducción. Hjalager, (2010) señala que en el turismo las barreras para innovar tienen que ver con el acercamiento al conocimiento como, por ejemplo patentes, a la difusión de la información y a la imitación.

Por otra parte, Hjalager, (2010) enfatiza que IP se refiere a las iniciativas que apuntan a la escalada de la eficiencia, la productividad y el flujo. La inversión en tecnología se convierte en la principal fuente, y generaría mayor eficiencia si se combina con otra estratégica y medidas de gestión, tales como el desarrollo de competencias y la gestión de recursos humanos. 
La IO de acuerdo con Jacob y Aguiló, (2008) estimula la productividad y la calidad de los servicios turísticos en virtud de que los cambios organizativos coadyuvan en la motivación de innovaciones para enfrentarse a nuevos desafíos y dificultades. En tanto Hjalager, (2010) dice que las innovaciones gerenciales, llevan a formas de organización distintas en donde la colaboración interna, la dirección y la capacitación de personal se conjuntan.

\section{METODOLOGÍA}

La investigación se desarrolló mediante el enfoque mixto. Se diseñaron dos instrumentos: el cuestionario y la entrevista semiestructurada. El cuestionario fue adaptado con base en el Manual de Oslo (OCDE, 2006), de esta manera en la primera parte se preguntó sobre información general de los establecimientos: la asociación a la que pertenecen, número de empleados que laboran, porcentaje de usuarios tanto nacionales como extranjeros que se hospedan, tipo de establecimiento por sus inversiones de capital, sean nacional o extranjero, si se realizan actividades innovadoras y sus tipos.

En la segunda parte se preguntó, primeramente, por la variable actividades de innovación y esta contiene nueve ítems; después por los factores que motivan las IP e IO y contiene las variables: competencia, demanda y mercado con 12 ítems; organización del lugar de trabajo con cinco ítems y, por último, otros factores con dos ítems. Además, se preguntó sobre los factores que obstaculizan la IP e 10 a través de las variables: de costo que contiene cinco ítems; vinculados al conocimiento con 16 ítems $y$, otras razones para no innovar con dos ítems.

El instrumento para la entrevista semiestructurada se diseñó considerando tres preguntas abiertas: ¿Existen actividades de innovación en la hotelería de Mazatlán?, ¿Qué factores motivan las IP e IO? y por último ¿Qué factores obstaculizan las IP e IO?

La muestra se determinó de una población de 40 hoteles integrados en asociaciones, 13 corresponde a establecimientos registrados en la Asociación de Hoteles y Moteles Tres Islas de Mazatlán (AHMTIM) y 27 a la Asociación de Hoteles y Empresas Turísticas de Mazatlán (AHETM) y como resultado fue estudiar a 11, distribuidos de manera proporcional en función a la asociación que pertenecen.

Además, se consideraron los criterios siguientes:

a) Los informantes seleccionados para el cuestionario fueron los gerentes generales de los establecimientos y para las entrevistas personajes directamente relacionados con la actividad: los presidentes de las dos asociaciones de hoteles y un experto en el tema turístico.

b) Ubicación geográfica: hoteles que se encuentran sobre la playa o que se separan de ésta mediante una calle.

c) Establecimientos integrados en alguna de las dos asociaciones.

d) Establecimientos con funcionamiento en el mercado superior a los tres años. 
Asimismo, se utilizan variables nominales para efectos de la evaluación y de comparación como la media aritmética, la cual indica el valor promedio y de frecuencia relativa. En el análisis entre variables e indicadores que lo integran, se busca identificar y explicar en función de los avances mostrados considerando que el desarrollo se define con la innovación.

\section{RESULTADOS}

Los hallazgos se presentan primeramente a partir del cuestionario y en segundo lugar se tienen los de las entrevistas semiestructuradas.

\subsection{HALLAZGOS A PARTIR DEL CUESTIONARIO}

Estos se presentan en la información general de establecimientos, las actividades de innovación, los factores que motivan la IP e 10 y por último los factores que obstaculizan innovar.

\subsubsection{Información general de los establecimientos}

Los datos de los hoteles informantes arrojaron los hallazgos siguientes: $45.5 \%$ pertenecen AHMTIM y 54.5 \% AHETM; la conformación de visitantes es de $85.6 \%$ nacionales y $14.4 \%$ internacionales y sus inversiones de capital son $90.9 \%$ nacionales y $9.1 \%$ extranjeros.

Los establecimientos informaron que $36.4 \%$ han realizado innovaciones de procesos y $54.5 \%$ innovaciones organizativas, las que se presentan exitosas en $90.9 \%$ de los casos y en curso $72.7 \%$.

\subsubsection{Actividades de innovación}

Los establecimientos señalaron, gráfica 1, que $100 \%$ planifican e introducen nuevos métodos de organización, $85.7 \%$ introdujeron, desarrollaron y ensayaron métodos de procesos, realizaron investigación y desarrollo y formación de capital humano, mientras que $28.6 \%$ adquirieron terrenos.

Gráfico 1. Actividades de innovación.

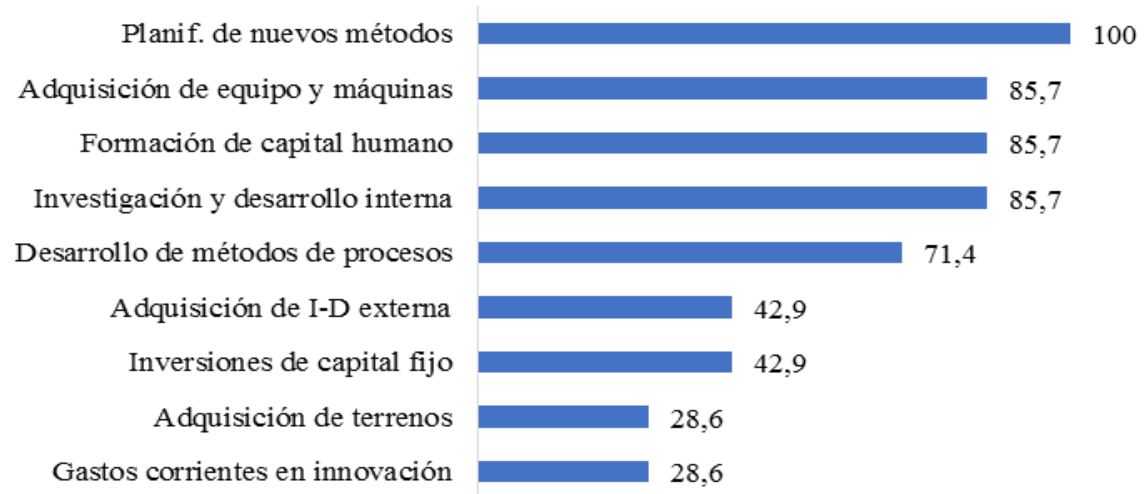

Fuente: elaboración propia. 


\subsubsection{Factores o efectos que motivan las innovaciones en la empresa hotelera}

Los hallazgos, tabla 1, presentaron tendencia como sigue:

Tabla 1. Factores y efectos que motivan la innovación. (Porcentajes)

\begin{tabular}{|l|c|c|}
\hline Factores y efectos & IP & IO \\
\hline Competencia, demanda y mercado (promedio) & 72.6 & 63.9 \\
\hline Organización del lugar de trabajo (promedio) & 77.1 & 70.0 \\
\hline Otros (promedio) & 92.9 & 66.7 \\
\hline
\end{tabular}

Fuente: elaboración propia.

El promedio $72.6 \%$ correspondió a la variable competencia, demanda y mercado en IP y 10 63.9\%. El promedio $77.1 \%$ de la variable organización del lugar de trabajo fue para la IP y 92.9\% para IO. El promedio de la variable otros fue $92.9 \%$ para la IP y $66.7 \%$ para la 10. $100 \%$ de los establecimientos señalaron que los factores que motivan la IP es reducir el impacto medioambiental o mejorar la sanidad y la seguridad, mientras que para la 10 este factor es $66.7 \%$.

\subsubsection{Factores que obstaculizan la innovación}

Los hallazgos, gráfico 2, presentan tendencia como sigue:

Gráfico 2. Factores que obstaculizan la innovación. (Porcentajes).

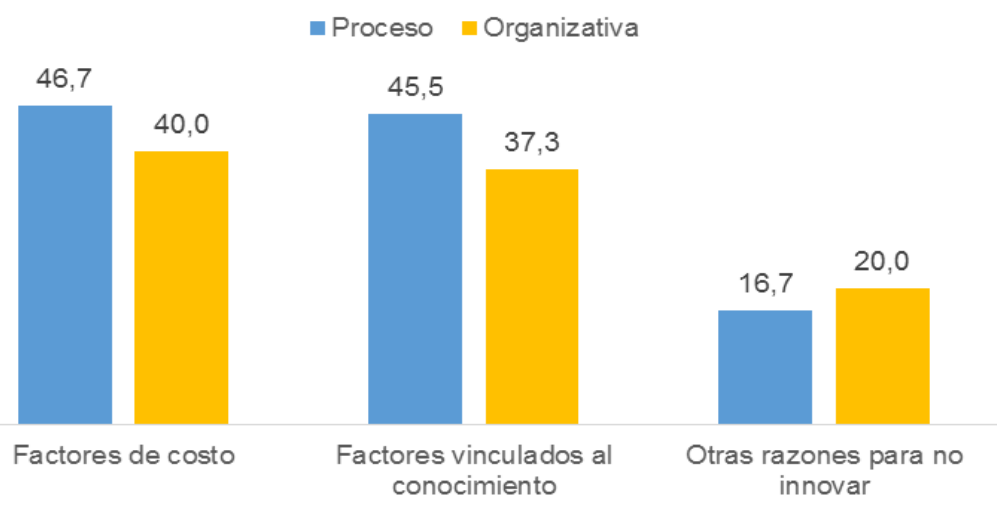

Fuente: elaboración propia.

El promedio $46.7 \%$ corresponde a la variable factores de costo para IP y $40 \%$ para IO. $83.3 \%$ de los establecimientos mencionaron riesgos percibidos como excesivos en la IP en cambio en la IO 60\%. En cambio, en capital de riesgo mencionan $16.7 \%$ para IP y $20 \%$ para la IO.

El promedio $45.6 \%$ representa la variable factores vinculados al conocimiento para IP y $37.3 \%$ para 10 . 


\section{$\begin{array}{llll}4.2 & \text { HALLAZGOS A PARTIR DE ENTREVISTAS }\end{array}$ SEMIESTRUCTURADAS}

Las categorías identificadas de acuerdo a cada pregunta son las siguientes:

¿Existen actividades de innovación en la hotelería de Mazatlán?

Actividades de innovación producto del esfuerzo individual ante la competencia. Esto de frente a una escasa vinculación del sector hotelero.

¿Qué factores motivan las IP e IO?

La inseguridad tractora de las actividades de innovación. Las dificultades de inseguridad por las que ha atravesado el destino ha llevado a innovar en simplificación de procesos, abaratamiento de costos, eficiente uso de materias primas esto se da mediante introducción de software y capacitación de recurso humano, principalmente.

Diferenciación mediante habilidades y equipamiento. Un área de oportunidad para los hoteles pequeños ha sido la actualización en habilidades y equipamiento ya que el precio no garantiza la selección del visitante.

La hotelería sustentable como práctica. Reconocimiento y adaptación de buenas prácticas que se realizan en otros destinos para reducir los costos de los consumos de servicios, operación y generación de residuos a través de una política ambiental de impacto en la variable competencia, demanda y mercado. En este proceso hubo una relación entre el Consejo para el Desarrollo Económico de Sinaloa (CODESIN, 2016) el Gobierno del estado de Sinaloa, la Asociación Civil Costas y Comunidades (CONSELVA) y las dos asociaciones de hoteles de Mazatlán de donde surge el proyecto Buenas Prácticas de Sustentabilidad de Administración y Operación Hotelera de Mazatlán.

¿Qué factores obstaculizan las IP e IO?

Insuficiencia en la inversión de infraestructura turística. La falta de inversión en infraestructura turística impide acelerar indicadores competitivo y de innovación.

\section{CONCLUCIONES}

Las IP e IO del sector hotelero son moderadas de acuerdo al resultado obtenido $36.4 \%$ y $54.5 \%$ respectivamente. Estas innovaciones como la introducción de nuevos métodos de apoyo a procesos y prácticas organizacionales, entre otras, estimulan la productividad y la calidad de los servicios turísticos Jacob y Aguiló (2008), convirtiéndose en ventajas competitivas que se transfieren al destino Mazatlán cuya tendencia es su reposicionamiento (Mathison et al. 2007) como destino turístico de sol y playa y en el que convergen otros segmentos (Reuniones y negocios, alternativo, cultural).

La experiencia de los actores se ha acumulado ante momentos difíciles como la inseguridad, la competencia y la demanda del visitantes, haciendo frente con las certificaciones de gestión (distintivos $\mathrm{H}$, distintivos $\mathrm{M}$, distintivo Verde), el mejoramiento de 
habilidades y la incorporación de tecnología, equipamiento (Weiermair, 2004) y (Hjalager, 2010) y adquisición de terrenos y el Proyecto buenas prácticas de sustentabilidad de administración y operación hotelera de Mazatlán (CODESIN, 2016).

En esta ruta hay advertencias, la innovación parte de un esfuerzo individual y no de una actividad vinculada. Hay una relación entre los actores para revisar el presente, pero no hay una vinculación para observar y analizar el futuro. El proyecto de buenas prácticas debe permear hacia otros subsectores de la actividad incluyendo al gobierno municipal que proporciona los servicios públicos mientras no se puede hablar de destino verde.

Las fuerzas impulsoras de la IP e IO competencia, demanda y mercado marcan una tendencia escasamente menor con relación a la organización del lugar del trabajo lo que es significativo ya que los factores reducir plazo de respuesta a las necesidades de los visitantes de frente a reducir costos en el diseño de productos se relaciona con lo mencionado por Hjalager (2010) la IP se refiere a las iniciativas que apuntan a la escalada de la eficiencia, la productividad y el flujo en donde la rapidez y mejora del servicio tienen sentido.

En relación con los factores que son obstáculos para la IP e IO la diferencia entre los de costo y los vinculados al conocimiento son mínimas, pero en los primeros es significativo el valor que se le da a los riesgos percibidos como excesivos esto tiene que ver con una cultura individualista para innovar que repercute en la institucionalización de la innovación.

Como conclusión general se tiene que la IP e IO de las empresas hoteleras contribuyen a fortalecer sus ventajas competitivas y también las del destino turístico Mazatlán, y con ello su reposicionamiento. La innovación se convierte en una ventaja competitiva.

Los elementos de la IP e IO en el destino Mazatlán se tienen en factores que motivan la innovación, destacando que marcan tendencia los de organización del lugar de trabajo para mejorar la comunicación e interacción en las distintas actividades de la empresa y aumentar la adaptabilidad a las distintas demandas de los visitantes y con ello la calidad del servicio (Jacob y Aguiló, 2008). Esto de frente a innovar por los riesgos percibidos como excesivos, obstáculo que se debe superar con un mayor conocimiento y vinculación del sector hotelero.

El enfoque mixto de investigación permitió integrar las visiones tanto de gerentes generales de hoteles, presidentes de asociación hoteleras y experto en tema turístico con resultados que dan cuenta de la empresa turística pero también de sus implicaciones al tomar decisiones con respecto a la innovación y su contribución al destino.

Por último, esta investigación permite orientar otras nuevas líneas de investigación que den cuenta desde el destino turístico la realidad de los liderazgos y sus visiones con respecto a la actividad, la gestión del conocimiento como orientadora para institucionalizar la innovación y la política turística ante la innovación. 


\section{REFERENCIAS BIBLIOGRÁFICAS}

Afuah, A. (1999). La dinámica de la innovación organizacional, el nuevo concepto para lograr ventajas competitivas y rentabilidad. México: Oxford.

CODESIN. (2016). Entrega de reconocimientos de hotelería sustentable en la zona sur. Recuperado de: <http://codesin.mx/news/entrega-codesin-reconocimientodistintivos- hoteleria\%20- sustentable/>.

Corma, F. (2011). Innovación, innovadores y empresa innovadora. España: Editorial Díaz de Santos.

Hall, M. y Williams, A. (2008). Tourism and Innovation. Routledge. USA: Tayor \& Francis Group.

Hjalager, A. M. (2010). A review of innovation research in tourism. Tourism Management. 31(2010):1-12. Recuperado de : $<$ https://www.academia.edu/5254430/Progress in Tourism Management A revi ew of innovation research in tourism/>.

Jacob, M. y Aguiló, E. (2008). La innovación en el sector turístico el caso de Baleares. Rotour/Revista de Ocio y Turismo. No. 1, 51-64. DOI: http://dx.doi.org/10.17979/rotur.2008.1.1.1225. [15 de septiembre de 2015].

Mathison, L., Gándara, J., Primera, C. y García, L. (2007). Innovación: Factor Clave para Lograr Ventajas Competitivas. Revista NEGOTIUM/Ciencias Gerenciales, 3(7). 4683. Recuperado de: <http://www.redalyc.org/articulo.oa?id=78230705/>. [20 de octubre de 2016].

Mohd, A. y Hilmi, M. (2012). Challenges and Outcome of Innovative Behavior: A Qualitative Study of Turism Related Entrepreneurs. Journal of Tecnology Managemen\&Innovation, 7(72), 131-14. Recuperado de: $<$ http://www.scielo.cl/scielo.php?script=sci arttext\&pid=S071827242012000200011/>. [15 de septiembre de 2016].

Nieves, J. y Segarra-Ciprés, M. (2015). Management innovation in the hotel industry. Tourism Management 46, 51-58. Recuperado de: http://dx.doi.org/10.1016/j.tourman.2014.06.002. [20 de octubre de 2016].

Organización para la Cooperación y el Desarrollo Económico. (2006). Manual de Oslo. España: Grupo Tragsa.

Pivcevic, S. y Garbin, D. (2012). Innovation activity in the hotel sector. the case of Croatia. Economic Research - Ekonomska Istrazivanja. 25, SE 1, 337-363. Recuperado de: <http://hrcak.srce.hr/103242?lang=en/>. [30 de septiembre de 2016].

Ritchie, J.R.B. y Crouch, G. I. (2011). The competitive destination a sustainable tourism perspective. UK: Cab International. 
Santamaría, A. y Barbosa, A. (2008). Ciclo turístico en Mazatlán. Fase de Exploracióninvolucramiento desarrollo (1970-1974). Estancamiento o crisis sistémica (19922004). Topofilia Revista Ar Arquitectura, Urbanismo y Ciencias Sociales Centro de Estudios de América del Norte, I(1). Recuperado de: $<$ http://148.228.173.140/topofiliaNew/assets/santamaria.pdf/ $>$. [20 de septiembre de 2016].

Secretaria de Turismo Federal. (2014). Agenda de competitividad del destino turístico de Mazatlán.

Secretaría de Turismo Federal (2015). Compendio Estadístico del turismo en México. Recuperado de: $\langle$ http://datatur.sectur.gob.mx/>. [25 de julio de 2016].

Vila, M., Enz, C. y Costa, G. (2012). Innovative practices in the Spanish hotel industry. Cornell Hospitality Quarterly, 53(1), 75-85. Cornell University, School of Hospitality Administration site. Recuperado de: $<$ http://scholarship.sha.cornell.edu/articles/204/>. [30 de marzo de 2016].

Weiermair, K. (2004). Product improvement or innovation: what is the key to success in tourism? En OECD innovation and growth in tourism: Conference Papers. Recuperado de: <http://www.oecd.org/cfe/tourism/34267947.pdf/>. [28 de junio de 2016]. 